Tritium Concentrations in Bees and Honey at Los Alamos National Laboratory: 1979-1996 
Edited by Hector Hinojosa, Group CIC-1

This report was prepared as an account of work sponsored by an agency of the United States Government. Neither The Regents of the University of Califormia, the United States

Government nor any agency thereof, nor any of their employees, makes any warranty, express or implied, or assumes any legal liability or responsibility for the accuracy, completeness, or usefulness of any information, apparatus, product, or process disclosed, or represents that its use would not infringe privately owned rights. Reference herein to any specific commercial product, process, or service by trade name, trademark, manufacturer, or otherwise, does not necessarily constitute or imply its endorsement, recommendation, or favoring by The Regents of the University of Califormia, the United States Government, or any agency thereof. The viezus and opinions of authors expressed herein do not necessarily state or reflect those of The Regents of the University of California, the United States Government, or any agency thereof. Los Alamos National Laboratory strongly supports academic freedom and a researcher's right to publish; as an institution, however, the Laboratory does not endorse the viewpoint of a publication or guarantee its technical correctness. 
UC-902 and UC-908 Issued: January 1997

Tritium Concentrations in Bees and Honey at Los Alamos National Laboratory: 1979-1996
P. R. Fresquez
D. R. Armstrong
L. H. Pratt

\section{Los Alamos \\ NATIONAL LABORATORY \\ Los Alamos, New Mexico 87545}


DISCLAIMER

Portions of this document may be illegible in electronic image products. Images are produced from the best available original document. 


\title{
TRITIUM CONCENTRATIONS IN BEES AND HONEY AT LOS ALAMOS NATIONAL LABORATORY: 1979-1996
}

by

P. R. Fresquez, D. R. Armstrong, and L. H. Pratt

\begin{abstract}
Honeybees are effective monitors of environmental pollution. The objective of this study was to summarize tritium $\left({ }^{3} \mathrm{H}\right)$ concentrations in bees and honey collected from within and around Los Alamos National Laboratory (LANL) over an 18-year period. Based on the long-term average, bees from nine out of eleven hives and honey from six out of eleven hives on LANL lands contained ${ }^{3} \mathrm{H}$ that was significantly higher $(p<0.05)$ than background. The highest average concentration of ${ }^{3} \mathrm{H}$ in bees $\left(435 \mathrm{pCi} \mathrm{mL}^{-1}\right)$ collected over the years was from LANL's Technical Area (TA) 54-a low-level radioactive waste disposal site (Area G). Similarily, the highest average concentration of ${ }^{3} \mathrm{H}$ in honey $\left(709 \mathrm{pCi} \mathrm{mL}^{-1}\right)$ was collected from a hive located near three ${ }^{3} \mathrm{H}$ storage ponds at LANL TA-53. The average concentrations of ${ }^{3} \mathrm{H}$ in bees and honey from background hives was $1.0 \mathrm{pCi} \mathrm{mL}^{-1}$ and $1.5 \mathrm{pCi}$ $\mathrm{mL}^{-1}$, respectively. Although the concentrations of ${ }^{3} \mathrm{H}$ in bees and honey from most LANL and perimeter (White Rock/Pajarito Acres) areas were significantly higher than background, most areas, with the exception of TA-53 and TA54 , generally exhibited decreasing ${ }^{3} \mathrm{H}$ concentrations over time.
\end{abstract}

\section{INTRODUCTION}

Honeybees are effective monitors of environmental pollution; they forage for pollen and nectar over a large area (e.g., 7 sq. $\mathrm{km}$ ) (Wallwork-Barber et al. 1982), accumulate contaminants from all four media (e.g., air, water, plants, and soil) (Bromenshenk et al. 1985), and return to a fixed location (the hive) for sampling (Simmons et al. 1990). The presence and distribution of pesticides (Anderson and Wojtas 
1986), polychlorinated biphenyls

(Morse et al. 1987), heavy metals

(Crane 1984, Jong and Morse 1977,

Free et al. 1983), and radioactivity

(Simmons et al. 1990, Hakonson and

Bostick 1976, and Kirkham and

Corey 1977) have all been assessed using honeybee colony networks. Hakonson and Bostick (1976), in particular, showed the usefulness of honeybee colonies for determining the presence of ${ }^{3} \mathrm{H},{ }^{137} \mathrm{Cs}$, and $\mathrm{Pu}$ in the environment within the Los Alamos National Laboratory (LANL) area. Of the three radionuclides studied, ${ }^{3} \mathrm{H}$ was most readily collected by the bees and transferred to the honey.

The objective of this study was to summarize ${ }^{3} \mathrm{H}$ concentrations in bees and honey collected from hives located within and around LANL over an 18-year period.

Results of other radionuclides $\left({ }^{57} \mathrm{Co}\right.$, ${ }^{60} \mathrm{Co},{ }^{152} \mathrm{Eu},{ }^{40} \mathrm{~K},{ }^{7} \mathrm{Be},{ }^{22} \mathrm{Na},{ }^{54} \mathrm{Mn}$, ${ }^{83} \mathrm{Rb},{ }^{137} \mathrm{Cs},{ }^{238} \mathrm{Pu},{ }^{239} \mathrm{Pu},{ }^{90} \mathrm{Sr},{ }^{241} \mathrm{Am}$, and ${ }^{\text {tot }} \mathrm{U}$ ) in honey collected from hives located around the perimeter of the Laboratory (Los Alamos and White Rock/Pajarito Acres, New Mexico) have been previously described (Fresquez et al., 1997).

\section{MATERIALS \& METHODS}

Over the course of this investigation, approximately 11 LANL, two perimeter (Los Alamos and White Rock/Pajarito Acres), and as many as five regional background areas (San Pedro, San Juan, Pojoaque, El Rancho, and/or Chimayo, New Mexico), were established for study (Figure 1). At each of these sites, two standard 1.7by $1.4-$ by 0.8 - $\mathrm{ft}$ Langstroth hives containing nine 0.5 - by $0.8-\mathrm{ft}$ frames were stocked with approximately 3 $\mathrm{lb}$ of Italian honeybees (Apis mellifera). Annually, around late August early September, approximately $500 \mathrm{~g}$ of forager and nurse bees and two frames of honey were placed into clean plastic bags, marked for identification, and transported to the Laboratory in locked ice chests. 


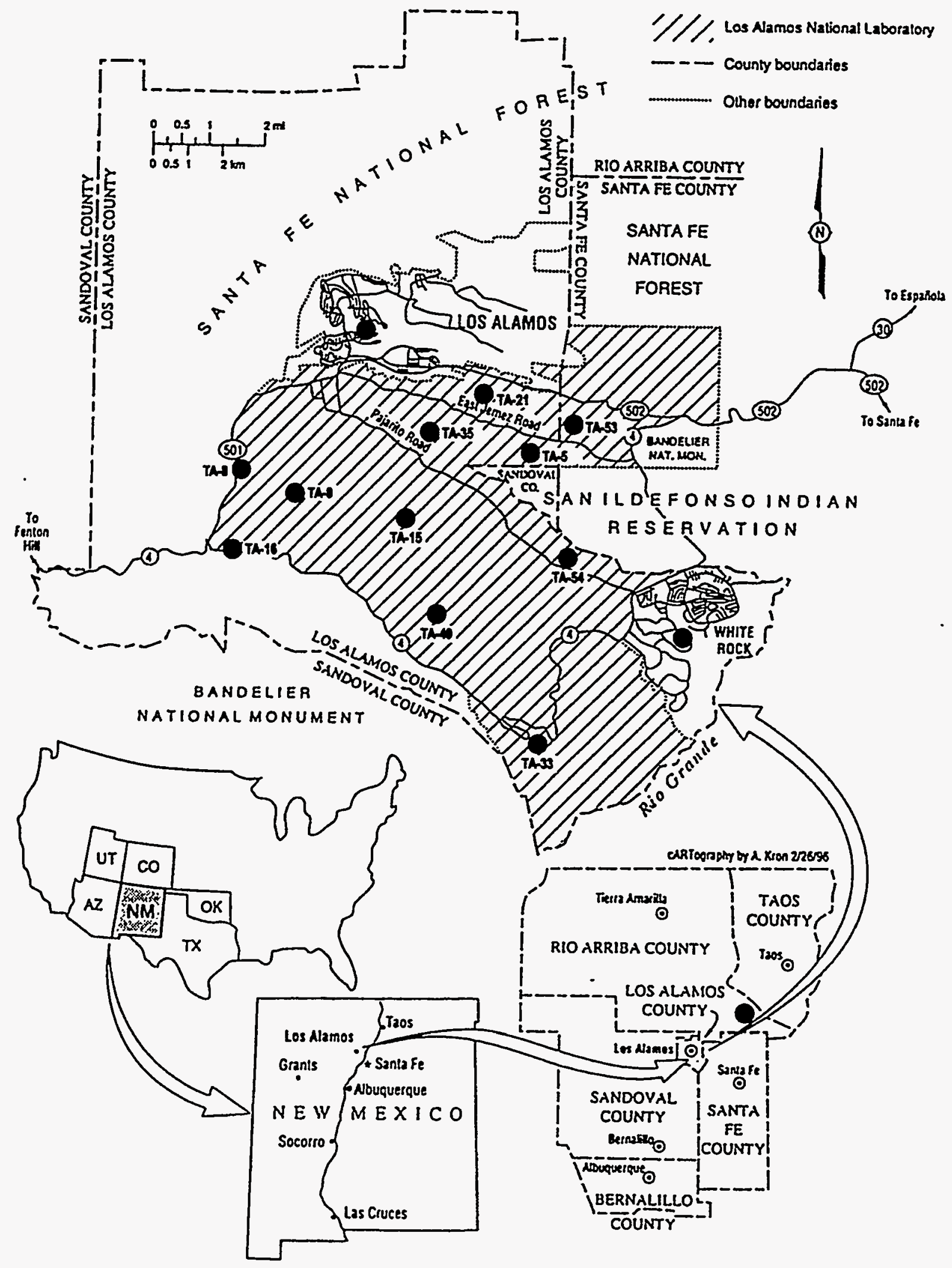

Beehive Locations

Figure 1. Locations of beehives on LANL, perimeter, and regional background areas. 
Bees from LANL and perimeter areas were collected from 1982 through 1993 and 1982 through 1996, respectively. Honey from LANL and perimeter areas was collected from 1979 through 1995 and 1979 through 1996, respectively.

At the Laboratory, $5 \mathrm{~mL}$ of moisture were distilled from each sample, mixed with $15 \mathrm{~mL}$ of a scintillation solution, and counted with a scintillation counter for 50 min. for ${ }^{3} \mathrm{H}$.

A nonparametric Wilcoxon Rank Sum test was used to assess differences in the arithmetic means of ${ }^{3} \mathrm{H}$ concentrations in bees and honey collected from LANL and perimeter stations versus regional background (all regional stations were combined) at the 0.01 and 0.05 probability level (Gilbert 1987). Pearson's correlation coefficients were used to compare ${ }^{3} \mathrm{H}$ concentrations in bees with ${ }^{3} \mathrm{H}$ concentrations in honey among the study sites. Also, a Mann-Kendall nonparametric test was used to evaluate trends in ${ }^{3} \mathrm{H}$ detected in bees and honey collected from LANL, perimeter, and regional background hives over time at the 0.05 probability level. The probability for the Mann-Kendall test was taken from Hollander and Wolfe (1973) in those cases with $<10$ data points and from the normal approximation in those cases with $>10$ data points. A positive value for the statistic indicated an upward trend while a negative value for the statistic indicated a downward trend (the direction indicated by the statistic was reported even when the test result was not significant).

\section{RESULTS \& DISCUSSION}

\section{a. Honeybees}

The upper limit background concentration (ULB) (i.e., the background mean plus two standard deviations) for ${ }^{3} \mathrm{H}$ in bees collected from regional background hives over the study period was 3.4 pCi mL ${ }^{-1}$ (Table 1). In general, ${ }^{3} \mathrm{H}$ in bees collected from LANL areas 
TABLE 1. Tritium (pCi mL ${ }^{-1}$ ) in bees from LANL, perimeter, and regional background areas between 1982 and 1996.

\begin{tabular}{|c|c|c|c|c|c|c|c|c|c|c|c|}
\hline & 1982 & 1986 & 1987 & 1988 & 1989 & 1990 & 1991 & 1992 & 1993 & 1996 & Mean \\
\hline \multicolumn{12}{|l|}{ LANL } \\
\hline \multirow[t]{2}{*}{ TA-5 } & 1 & 14.0 & 5.7 & 30.0 & 44.0 & 7.2 & 1.0 & 20.9 & 6.5 & & $16.2^{* *}$ \\
\hline & & $(4.0)^{2}$ & (1.4) & $(6.0)$ & (8.0) & (1.6) & $(0.6)$ & (2.8) & (1.6) & & $(29.3)^{3}$ \\
\hline \multirow[t]{2}{*}{ TA-8 } & 1.8 & 7.7 & 4.7 & 0.7 & 1.8 & 3.5 & 0.5 & 14.6 & 0.6 & & $4.0^{*}$ \\
\hline & & (1.8) & (1.2) & $(0.6)$ & $(0.8)$ & $(1.0)$ & $(0.6)$ & (2.4) & $(0.6)$ & & (9.3) \\
\hline \multirow[t]{2}{*}{ TA-9 } & & 12.0 & 1.6 & 0.3 & 1.5 & 5.7 & 0.7 & 1.1 & 0.6 & & 2.9 \\
\hline & & $(2.0)$ & (1.2) & $(0.6)$ & $(0.6)$ & $(1.4)$ & $(0.6)$ & $(0.6)$ & $(0.6)$ & & $(8.1)$ \\
\hline \multirow[t]{2}{*}{ TA-15 } & & 5.3 & 2.2 & 2.3 & 780.0 & 2.4 & 5.3 & 13.1 & 6.9 & & $102.2^{* *}$ \\
\hline & & (1.4) & $(0.8)$ & $(0.8)$ & $(160.0)$ & $(0.8)$ & $(1.1$ & $(2.2)$ & $(1.6)$ & & $(547.8)$ \\
\hline \multirow[t]{2}{*}{ TA-16 } & & & 1.1 & 6.8 & 5.2 & 4.4 & 0.4 & 0.3 & 1.1 & & 2.8 \\
\hline & & & $(0.8)$ & $(1.6)$ & $(1.2)$ & $(1.0)$ & $(0.6)$ & $(0.6)$ & (0.6) & & $(5.3)$ \\
\hline \multirow[t]{2}{*}{ TA-21 } & 3.6 & & 23.0 & 6.7 & 18.0 & 19.0 & 8.2 & 16.1 & 4.9 & & $12.4^{* * *}$ \\
\hline & $(2.4)$ & & (4.0) & (1.6) & $(4.0)$ & $(4.0)$ & (1.6) & $(2.4)$ & (1.4) & & $(14.8)$ \\
\hline \multirow[t]{2}{*}{ TA-33 } & 35.0 & 8.7 & 30.0 & 4.9 & 430.0 & 47.0 & 14.1 & 13.5 & 9.9 & & $65.9 * *$ \\
\hline & $(23.1)$ & $(2.0)$ & $(6.0)$ & $(1.2)$ & $(80.0)$ & $(10.0)$ & $(2.8)$ & $(2.2)$ & $(2.0)$ & & $(274.5)$ \\
\hline \multirow[t]{2}{*}{ TA-35 } & & 21.0 & 3.6 & 63.0 & 190.0 & 25.0 & 1.8 & 1.7 & 15.6 & & $40.2^{* *}$ \\
\hline & & $(4.0)$ & $(1.0)$ & $(12.0)$ & $(4.0)$ & $(6.0)$ & $(0.6)$ & $(0.8)$ & (2.4) & & (127.5) \\
\hline \multirow[t]{2}{*}{ TA-49 } & & & 2.0 & 0.6 & 8.6 & 5.6 & 0.9 & 1.6 & 0.8 & & $2.9 *$ \\
\hline & & & $(0.8)$ & $(0.6)$ & $(2.0)$ & $(1.4)$ & $(0.6)$ & $(0.8)$ & $(0.6)$ & & $(6.1)$ \\
\hline \multirow[t]{2}{*}{ TA-53 } & 15.0 & 6.1 & 16.0 & 110.0 & 3300.0 & 55.0 & 4.9 & 21.7 & 245.7 & & $419.4^{* *}$ \\
\hline & $(9.9)$ & (1.6) & $(4.0)$ & $(20.0)$ & $(600.0)$ & $(12.0)$ & $(1.0)$ & $(2.8)$ & (11.4) & & $(2,166.1)$ \\
\hline \multirow[t]{2}{*}{ TA-54 } & 38.0 & & 260.0 & 130.0 & 1800.0 & 760.0 & 24.1 & 411.8 & 54.4 & & $434.8 * *$ \\
\hline & $(25.0)$ & & $(60.0)$ & $(20.0)$ & $(400.0)$ & $(160.0)$ & $(4.8)$ & $(16.2)$ & (4.6) & & $(1,210.0)$ \\
\hline \multicolumn{12}{|c|}{ PERIMETER } \\
\hline \multirow[t]{4}{*}{$\mathrm{LA}^{4}$} & 1.8 & & & & 0.1 & & & & 0.6 & -0.3 & 0.7 \\
\hline & $(1.2)$ & & & & $(0.6)$ & & & & $(0.6)$ & $(0.3)$ & $(1.6)$ \\
\hline & & & & & & & & & & 0.7 & \\
\hline & & & & & & & & & & $(0.3)$ & \\
\hline \multirow[t]{2}{*}{ WR/PA } & 11.0 & 4.6 & 3.1 & 10.0 & & & & & 34.6 & -0.1 & $10.5^{*}$ \\
\hline & $(7.3)$ & $(1.2)$ & $(1.0)$ & (2.0) & & & & & (3.6) & $(0.3)$ & $(25.0)$ \\
\hline \multicolumn{12}{|c|}{ BACKGROUND } \\
\hline & 0.7 & 3.7 & 2.8 & 0.2 & 0.4 & 1.0 & 0.6 & 0.2 & 0.6 & 0.2 & 1.0 \\
\hline & $(0.0)$ & (3.7) & $(3.0)$ & $(0.6)$ & $(0.5)$ & $(0.9)$ & $(0.3)$ & $(0.1)$ & $(0.4)$ & $(0.3)$ & $(2.4)$ \\
\hline
\end{tabular}

Analysis not performed or lost in analysis.

${ }^{2}( \pm 2$ counting uncertainty); values are the uncertainty in the analytical results at the $95 \%$ confidence level.

${ }^{3}( \pm 2$ standard deviation).

${ }^{4} \mathrm{LA}=$ Los Alamos and WR/PA=White Rock/Pajarito Acres

* and ** denotes significantly different from regional background at the 0.05 and 0.01 level using a Wilcoxcon Rank Sum Test, respectively. 
from 1982 through 1993 ranged in concentration from 0.30 to $3,300.00$ $\mathrm{pCi} \mathrm{mL}^{-1}$. Most hives on LANL lands in almost every year contained bees with ${ }^{3} \mathrm{H}$ above the ULB concentration(s). The highest concentrations of ${ }^{3} \mathrm{H}$ in bees year after year were consistently detected from hives located at Technical Area (TA) 53, the Los Alamos Neutron Scattering Center (LANSCE), and at TA-54, the Laboratory's active lowlevel waste burial site (Area G). Operations at TA-53 include the use of a high-energy linear particle accelerator, which, upon contact with the atmosphere, converts water vapor into ${ }^{3} \mathrm{HTO}$ (tritiated water vapor); and, probably more importantly, there are three radioactive storage ponds on site that contain ${ }^{3} \mathrm{H}$ (EPG 1995). Similarly, TA-54 (Area G) houses shafts that contain ${ }^{3} \mathrm{H}$ contaminated wastes, and several studies have confirmed the presence of ${ }^{3} \mathrm{H}$ in several environmental matrices (small mammals, soils, and vegetation) above that normally detected in materials collected from background locations (Biggs et al. 1995, Fresquez et al. 1996a, EPG 1995).

Tritium in bees collected from hives located along perimeter areas-Los Alamos and White Rock/Pajarito Acres-ranged in concentrations from $-0.3 \mathrm{pCi} \mathrm{mL} \mathrm{m}^{-1}$ (1996) to $1.8 \mathrm{pCi} \mathrm{mL}^{-1}$ (1982) and from $-0.1 \mathrm{pCi} \mathrm{mL}^{-1}$ (1996) to 34.6 pCi mL $\mathrm{mL}^{-1}$ (1993), respectively. Based on the average concentration of ${ }^{3} \mathrm{H}$ over the years, bees collected from LANL technical areas-TA-5, TA-8, TA-15, TA-21, TA-33, TA-35, TA49, TA-53, TA-54 and White Rock/Pajarito Acres had significantly higher $(p \quad<0.01$ and 0.05$){ }^{3} \mathrm{H}$ concentrations than bees collected from regional background locations. These data correlate well with those of Hakonson and Bostick (1976), who showed that ${ }^{3} \mathrm{H}$ concentrations in bees from three canyon liquid waste disposal areas within LANL increased from initial values of $<1.0$ $\mathrm{pCi} \mathrm{mL}^{-1}$ to as much as $9,200 \mathrm{pCi}$ 
TABLE 2. Results of the Mann-Kendall Nonparametric Test for ${ }^{3} \mathrm{H}$ Trends in Bees and Honey from LANL, Perimeter, and Regional Background.

\begin{tabular}{lll}
\hline Location & \multicolumn{1}{c}{ Bees } & \multicolumn{1}{c}{ Honey } \\
\hline LANL & 0.36 (Down) & 0.00 (Down)** $^{*}$ \\
TA-5 & 0.27 (Down) & 0.00 (Down)** $^{*}$ \\
TA-8 & 0.09 (Down) & 0.02 (Down)* \\
TA-9 & 0.89 (Up) & 0.03 (Down)* \\
TA-15 & 0.09 (Down) & 0.05 (Down)* \\
TA-16 & 0.45 (Down) & 0.55 (Up) \\
TA-21 & 0.30 (Down) & 0.00 (Down)** \\
TA-33 & 0.19 (Down) & 0.00 (Down)** \\
TA-35 & 0.27 (Down) & 0.13 (Down) \\
TA-49 & 0.83 (Up) & 0.95 (Up) \\
TA-53 & 0.50 (No Trend) & 1.00 (Up) \\
TA-54 & & \\
Perimeter & 0.37 (Down) & 0.09 (Down) \\
LA & 0.35 (Down) & 0.05 (Down)* \\
WR/PA & 0.05 (Down)* & 0.00 (Down) \\
Background & TA
\end{tabular}

${ }^{1} \mathrm{LA}=$ Los Alamos and WR/PA=White Rock/Pajarito Acres

$*$ and ${ }^{* *}$ denotes significance at the 0.05 and 0.01 probability level, respectively.

$\mathrm{mL}^{-1}$. Although the average concentrations of ${ }^{3} \mathrm{H}$ in bees from most LANL hives over the years were significantly higher than background, most areas, with the exception of TA-15 and TA-53, exhibited downward trends (Table 2). None of the trend directions, with the exception of background, were statistically significant, however.

\section{b. Honey}

The ULB concentration for ${ }^{3} \mathrm{H}$ in honey collected from regional hives over the 18-year period was 5.0 pCi $\mathrm{mL}^{-1}$ (Table 3). Tritium in honey collected from LANL areas from 1979 through 1995 generally ranged in concentration from -0.2 to $7,600 \mathrm{pCi} \mathrm{mL}^{-1}$. Again, ${ }^{3} \mathrm{H}$ concentrations in honey from most LANL hives and in most years were 
TABLE 3. Tritium (pCi mL $\mathrm{mL}^{-1}$ ) in honey from LANL, perimeter, and regional background areas between 1979 and 1996.

\begin{tabular}{|c|c|c|c|c|c|c|c|c|c|c|c|c|c|c|c|c|c|c|c|}
\hline & 1979 & 1980 & 1981 & 1982 & 1983 & 1984 & 1985 & 1986 & 1987 & 1988 & 1989 & 1990 & 1991 & 1992 & 1993 & 1994 & 1995 & 1996 & Mean \\
\hline \multicolumn{20}{|l|}{$\overline{\text { LANL }}$} \\
\hline TA-5 & $\begin{array}{c}11.8 \\
(7.8)^{2}\end{array}$ & $\begin{array}{c}27.4 \\
(18.1)\end{array}$ & $\begin{array}{l}13.6 \\
(9.0)\end{array}$ & $\begin{array}{c}7.2 \\
(4.8)\end{array}$ & 1 & $\begin{array}{l}12.0 \\
(7.9)\end{array}$ & $\begin{array}{l}10.0 \\
(2.0)\end{array}$ & $\begin{array}{c}8.4 \\
(2.0)\end{array}$ & $\begin{array}{c}7.7 \\
(5.1)\end{array}$ & $\begin{array}{c}1.0 \\
(0.6)\end{array}$ & $\begin{array}{c}1.6 \\
(0.8)\end{array}$ & $\begin{array}{c}4.9 \\
(0.6)\end{array}$ & $\begin{array}{c}0.1 \\
(0.6)\end{array}$ & $\begin{array}{c}0.8 \\
(0.6)\end{array}$ & $\begin{array}{c}0.6 \\
(0.6)\end{array}$ & $\begin{array}{l}-0.2 \\
(0.6)\end{array}$ & $\begin{array}{c}0.2 \\
(0.6)\end{array}$ & & $\begin{array}{c}6.7^{*} \\
(14.7)^{3}\end{array}$ \\
\hline TA-8 & & & & & $\begin{array}{c}7.7 \\
(5.1)\end{array}$ & $\begin{array}{c}4.8 \\
(3.2)\end{array}$ & $\begin{array}{c}59.0 \\
(12.0)\end{array}$ & $\begin{array}{c}0.4 \\
(0.8)\end{array}$ & $\begin{array}{c}5.9 \\
(3.9)\end{array}$ & $\begin{array}{c}1.6 \\
(0.6)\end{array}$ & $\begin{array}{c}2.6 \\
(0.8)\end{array}$ & $\begin{array}{c}0.8 \\
(0.6)\end{array}$ & $\begin{array}{c}0.4 \\
(0.6)\end{array}$ & $\begin{array}{c}0.5 \\
(0.6)\end{array}$ & & $\begin{array}{c}0.1 \\
(0.6)\end{array}$ & $\begin{array}{c}0.1 \\
(0.6)\end{array}$ & & $\begin{array}{c}7.0 \\
(33.2)\end{array}$ \\
\hline TA-9 & & & & & & $\begin{array}{c}1.7 \\
(1.1)\end{array}$ & $\begin{array}{l}13.0 \\
(2.0)\end{array}$ & $\begin{array}{c}4.5 \\
(0.8)\end{array}$ & $\begin{array}{c}1.0 \\
(0.7)\end{array}$ & $\begin{array}{c}0.1 \\
(0.6)\end{array}$ & $\begin{array}{c}1.4 \\
(0.6)\end{array}$ & $\begin{array}{c}0.8 \\
(0.6)\end{array}$ & $\begin{array}{c}0.2 \\
(0.6)\end{array}$ & $\begin{array}{l}29.1 \\
(3.4)\end{array}$ & $\begin{array}{l}-0.4 \\
(0.6)\end{array}$ & $\begin{array}{c}0.7 \\
(0.7)\end{array}$ & $\begin{array}{c}0.0 \\
(0.6)\end{array}$ & & $\begin{array}{c}4.3 \\
(17.2)\end{array}$ \\
\hline TA-15 & & & & & & $\begin{array}{c}4.2 \\
(2.8)\end{array}$ & $\begin{array}{l}26.0 \\
(6.0)\end{array}$ & $\begin{array}{c}4.3 \\
(1.2)\end{array}$ & $\begin{array}{c}0.5 \\
(0.6)\end{array}$ & $\begin{array}{c}0.6 \\
(0.6)\end{array}$ & $\begin{array}{c}3.0 \\
(1.0)\end{array}$ & $\begin{array}{c}1.0 \\
(0.6)\end{array}$ & $\begin{array}{c}5.4 \\
(0.6)\end{array}$ & $\begin{array}{c}1.2 \\
(0.8)\end{array}$ & $\begin{array}{c}0.6 \\
(0.6)\end{array}$ & $\begin{array}{l}-0.2 \\
(0.6)\end{array}$ & $\begin{array}{c}0.5 \\
(0.8)\end{array}$ & & $\begin{array}{c}4.0 \\
(14.4)\end{array}$ \\
\hline TA-16 & $\begin{array}{c}2.8 \\
(1.8)\end{array}$ & $\begin{array}{c}5.2 \\
(3.4)\end{array}$ & $\begin{array}{c}3.1 \\
(2.0)\end{array}$ & $\begin{array}{l}11.0 \\
(7.3)\end{array}$ & & & & & $\begin{array}{c}0.0 \\
(0.6)\end{array}$ & $\begin{array}{c}0.5 \\
(0.6)\end{array}$ & $\begin{array}{c}0.3 \\
(0.6)\end{array}$ & $\begin{array}{c}0.5 \\
(1.2)\end{array}$ & $\begin{array}{c}0.7 \\
(0.6)\end{array}$ & $\begin{array}{c}1.5 \\
(0.8)\end{array}$ & $\begin{array}{c}0.1 \\
(0.6)\end{array}$ & $\begin{array}{l}-0.3 \\
(0.6)\end{array}$ & $\begin{array}{c}0.6 \\
(0.6)\end{array}$ & & $\begin{array}{c}2.0 \\
(6.3)\end{array}$ \\
\hline TA-21 & $\begin{array}{c}5.8 \\
(3.8)\end{array}$ & $\begin{array}{c}5.6 \\
(3.7)\end{array}$ & $\begin{array}{c}18.2 \\
(12.0)\end{array}$ & $\begin{array}{c}9.0 \\
(6.0)\end{array}$ & $\begin{array}{c}81.0 \\
(53.5)\end{array}$ & $\begin{array}{c}29.0 \\
(19.1)\end{array}$ & $\begin{array}{r}6200 \\
(1200)\end{array}$ & $\begin{array}{c}7.5 \\
(1.8)\end{array}$ & $\begin{array}{l}14.0 \\
(9.2)\end{array}$ & $\begin{array}{c}3.9 \\
(1.0)\end{array}$ & $\begin{array}{l}31.0 \\
(6.0)\end{array}$ & $\begin{array}{l}110.0 \\
(20.0)\end{array}$ & $\begin{array}{c}9.1 \\
(1.8)\end{array}$ & $\begin{array}{l}49.9 \\
(5.5)\end{array}$ & $\begin{array}{l}12.0 \\
(2.2)\end{array}$ & $\begin{array}{c}2.0 \\
(0.8)\end{array}$ & $\begin{array}{c}9.9 \\
(2.0)\end{array}$ & & $\begin{array}{c}388.1^{* *} \\
(2996)\end{array}$ \\
\hline TA-33 & $\begin{array}{l}579.0 \\
(382)\end{array}$ & $\begin{array}{l}207.0 \\
(137)\end{array}$ & $\begin{array}{l}156.0 \\
(103)\end{array}$ & $\begin{array}{c}92.5 \\
(61.1)\end{array}$ & $\begin{array}{c}73.0 \\
(48.2)\end{array}$ & $\begin{array}{c}99.0 \\
(65.3)\end{array}$ & $\begin{array}{c}67.0 \\
(14.0)\end{array}$ & $\begin{array}{l}33.0 \\
(6.0)\end{array}$ & $\begin{array}{l}14.0 \\
(9.2)\end{array}$ & $\begin{array}{l}38.0 \\
(8.0)\end{array}$ & $\begin{array}{c}55.0 \\
(12.0)\end{array}$ & $\begin{array}{l}240.0 \\
(40.0)\end{array}$ & $\begin{array}{l}12.4 \\
(0.7)\end{array}$ & $\begin{array}{l}25.1 \\
(3.0)\end{array}$ & $\begin{array}{l}-0.2 \\
(0.6)\end{array}$ & $\begin{array}{l}21.3 \\
(2.8)\end{array}$ & $\begin{array}{c}5.2 \\
(1.4)\end{array}$ & . & $\begin{array}{l}101.0^{* *} \\
(283.6)\end{array}$ \\
\hline TA-35 & $\begin{array}{c}26.7 \\
(17.6)\end{array}$ & $\begin{array}{c}17.9 \\
(11.8)\end{array}$ & $\begin{array}{c}63.5 \\
(41.2)\end{array}$ & $\begin{array}{c}17.6 \\
(11.6)\end{array}$ & $\begin{array}{c}31.0 \\
(20.5)\end{array}$ & $\begin{array}{l}12.0 \\
(7.9)\end{array}$ & $\begin{array}{c}73.0 \\
(14.0)\end{array}$ & $\begin{array}{c}8.4 \\
(2.0)\end{array}$ & $\begin{array}{l}11.0 \\
(7.3)\end{array}$ & $\begin{array}{c}1.3 \\
(0.6)\end{array}$ & $\begin{array}{c}7.1 \\
(1.6)\end{array}$ & $\begin{array}{c}9.1 \\
(2.0)\end{array}$ & $\begin{array}{c}1.8 \\
(0.6)\end{array}$ & $\begin{array}{c}4.3 \\
(0.6)\end{array}$ & $\begin{array}{c}2.1 \\
(0.8)\end{array}$ & $\begin{array}{c}0.6 \\
(0.6)\end{array}$ & $\begin{array}{c}0.6 \\
(0.8)\end{array}$ & & $\begin{array}{l}16.9 * * \\
(42.7)\end{array}$ \\
\hline TA-49 & & & & & & & & & $\begin{array}{c}2.2 \\
(1.5)\end{array}$ & $\begin{array}{c}1.1 \\
(0.6)\end{array}$ & $\begin{array}{c}7.1 \\
(1.6)\end{array}$ & $\begin{array}{c}1.3 \\
(0.6)\end{array}$ & $\begin{array}{c}0.1 \\
(0.6)\end{array}$ & $\begin{array}{c}2.5 \\
(1.0)\end{array}$ & $\begin{array}{c}0.5 \\
(0.6)\end{array}$ & $\begin{array}{c}0.3 \\
(0.6)\end{array}$ & & & $\begin{array}{c}1.9 \\
(4.6)\end{array}$ \\
\hline TA-53 & & & & $\begin{array}{l}11.2 \\
(7.4)\end{array}$ & $\begin{array}{c}9.8 \\
(6.5)\end{array}$ & $\begin{array}{c}50.0 \\
(33.0)\end{array}$ & $\begin{array}{c}7600 \\
(1600)\end{array}$ & $\begin{array}{c}0.1 \\
(0.2)\end{array}$ & $\begin{array}{c}65.0 \\
(42.9)\end{array}$ & $\begin{array}{c}61.0 \\
(12.0)\end{array}$ & $\begin{array}{c}74.0 \\
(16.0)\end{array}$ & $\begin{array}{l}420.0 \\
(80.0)\end{array}$ & $\begin{array}{l}6.40 \\
(1.2)\end{array}$ & $\begin{array}{l}32.7 \\
(3.6)\end{array}$ & $\begin{array}{l}117.9 \\
(7.2)\end{array}$ & $\begin{array}{l}1300 \\
(1.0)\end{array}$ & $\begin{array}{l}179.0 \\
(34.0)\end{array}$ & & $\begin{array}{c}709.1^{* *} \\
(4024)\end{array}$ \\
\hline TA-54 & $\begin{array}{c}9.6 \\
(6.3)\end{array}$ & $\begin{array}{c}21.4 \\
(14.1)\end{array}$ & $\begin{array}{c}27.0 \\
(17.8)\end{array}$ & $\begin{array}{c}29.4 \\
(19.4)\end{array}$ & $\begin{array}{c}29.0 \\
(19.1)\end{array}$ & $\begin{array}{c}37.0 \\
(24.4)\end{array}$ & & & $\begin{array}{c}92.0 \\
(60.7)\end{array}$ & $\begin{array}{c}0.2 \\
(0.6)\end{array}$ & $\begin{array}{l}370.0 \\
(80.0)\end{array}$ & $\begin{array}{c}54.0 \\
(10.0)\end{array}$ & $\begin{array}{c}95.3 \\
(16.0)\end{array}$ & $\begin{array}{l}94.7 \\
(6.4)\end{array}$ & $\begin{array}{l}238.0 \\
(11.0)\end{array}$ & $\begin{array}{l}101.7 \\
(6.6)\end{array}$ & & & $\begin{array}{l}85.6 * * \\
(203.8)\end{array}$ \\
\hline \multicolumn{20}{|c|}{ PERIMETER } \\
\hline$L A^{5}$ & $\begin{array}{c}3.6 \\
(2.4)\end{array}$ & $\begin{array}{c}4.0 \\
(2.6)\end{array}$ & $\begin{array}{l}12.7 \\
(8.4)\end{array}$ & $\begin{array}{l}12.3 \\
(8.1)\end{array}$ & $\begin{array}{c}0.2 \\
(0.1)\end{array}$ & & $\begin{array}{c}860.0 \\
(180.0)\end{array}$ & & & & $\begin{array}{c}0.1 \\
(0.6)\end{array}$ & & & & $\begin{array}{c}0.3 \\
(0.6)\end{array}$ & $\begin{array}{c}0.2 \\
(0.6)\end{array}$ & $\begin{array}{c}0.0 \\
(0.6)\end{array}$ & $\begin{array}{c}0.4 \\
(0.3) \\
0.0 \\
(0.3) \\
1.3 \\
(0.4)\end{array}$ & $\begin{array}{c}81.3^{4} \\
(516.6)\end{array}$ \\
\hline $\begin{array}{l}\text { WR/ } \\
\text { PA }\end{array}$ & $\begin{array}{r}10.5 \\
(6.9)\end{array}$ & $\begin{array}{c}7.9 \\
(5.2)\end{array}$ & & $\begin{array}{c}3.2 \\
(2.1)\end{array}$ & $\begin{array}{c}4.9 \\
(3.2)\end{array}$ & $\begin{array}{c}4.0 \\
(2.6)\end{array}$ & $\begin{array}{c}9.0 \\
(2.0)\end{array}$ & $\begin{array}{c}2.3 \\
(0.8)\end{array}$ & $\begin{array}{c}20.0 \\
(13.2)\end{array}$ & $\begin{array}{c}0.2 \\
(0.6)\end{array}$ & & & & & $\begin{array}{l}37.3 \\
(8.6)\end{array}$ & $\begin{array}{c}2.4 \\
(1.0)\end{array}$ & $\begin{array}{l}-0.2 \\
(0.6)\end{array}$ & $\begin{array}{c}0.0 \\
(0.3)\end{array}$ & $\begin{array}{c}7.8^{*} \\
(20.9)\end{array}$ \\
\hline \multicolumn{20}{|c|}{ BACKGROUND $^{3}$} \\
\hline & $\begin{array}{c}0.6 \\
(0.0)^{4}\end{array}$ & $\begin{array}{c}3.0 \\
(0.0)\end{array}$ & $\begin{array}{c}6.3 \\
(0.0)\end{array}$ & $\begin{array}{r}1.3 \\
(0.0) \\
\end{array}$ & $\begin{array}{c}4.0 \\
(2.4)\end{array}$ & $\begin{array}{r}2.5 \\
(4.7) \\
\end{array}$ & $\begin{array}{c}1.6 \\
(3.7) \\
\end{array}$ & $\begin{array}{c}0.9 \\
(1.6) \\
\end{array}$ & $\begin{array}{r}3.8 \\
(6.0) \\
\end{array}$ & $\begin{array}{c}0.8 \\
(1.4) \\
\end{array}$ & $\begin{array}{c}0.3 \\
(0.6) \\
\end{array}$ & $\begin{array}{r}1.0 \\
(2.3) \\
\end{array}$ & $\begin{array}{c}0.1 \\
(0.4)\end{array}$ & $\begin{array}{c}0.4 \\
(0.5) \\
\end{array}$ & $\begin{array}{c}0.1 \\
(0.8) \\
\end{array}$ & $\begin{array}{c}-0.1 \\
(0.5) \\
\end{array}$ & $\begin{array}{c}0.1 \\
(0.6) \\
\end{array}$ & $\begin{array}{r}0.2 \\
(0.3) \\
\end{array}$ & $\begin{array}{r}1.5 \\
(3.5) \\
\end{array}$ \\
\hline
\end{tabular}

'Analysis not performed or lost in analysis. ${ }^{2} \pm 2$ counting uncertainty). ${ }^{3}\left( \pm 2\right.$ standard deviation). ${ }^{4}$ The average without the abnormally high 1985 result $=3.1$ ( \pm 9.7 [two sigma]). ${ }^{5} \mathrm{LA}=\mathrm{Los}$ Alamos and WR/PA $=$ White Rock/Pajarito Acres

* and ** denotes significantly different from regional background at the 0.05 and 0.01 level using a Wilcoxon Rank Sum Test, respectively. 
greater than the respective yearly ULB concentration(s); and, particularly at TA-53 (LANSCE). Concentrations of ${ }^{3} \mathrm{H}$ in honey collected from perimeter locationsLos Alamos and White Rock/Pajarito Acres-ranged from $0.0 \mathrm{pCi} \mathrm{mL}$ (1995) to $860 \mathrm{pCi} \mathrm{mL} \mathrm{m}^{-1}$ (1985) and from $-0.2 \mathrm{pCi} \mathrm{mL} L^{-1}$ (1995) to $37 \mathrm{pCi}$ $\mathrm{mL}^{-1}$ (1993) respectively. Based on the overall average, the concentrations of ${ }^{3} \mathrm{H}$ in honey collected from hives located at TA-5, TA-21, TA-33, TA-35, TA-53, TA54, and White Rock/Pajarito Acres were significantly higher $(p<0.01$ and 0.05 ) than background. The higher average concentration of ${ }^{3} \mathrm{H}$ in honey from Los Alamos as compared to White Rock/Pajarito Acres was the result of one very high ${ }^{3} \mathrm{H}$ result $\left(860 \mathrm{pCi} \mathrm{mL}^{-1}\right)$ in 1985 , when the Laboratory released $8,638 \mathrm{Ci}$ of ${ }^{3} \mathrm{H}$ to the atmosphere and $76,850 \mathrm{mCi}$ of ${ }^{3} \mathrm{H}$ to the canyons as liquid effluents (EPG 1986). Since these release points were closer to the Los Alamos townsite than to the White
Rock/Pajarito Acres townsite, Los Alamos was mostly affected. The average concentration of ${ }^{3} \mathrm{H}$ in honey from the Los Alamos townsite without the abnormally high $1985^{3} \mathrm{H}$ value would be only $3.1 \mathrm{pCi} / \mathrm{mL}$.

Although most hives on LANL lands and perimeter areas contained ${ }^{3} \mathrm{H}$ concentrations in honey that were significantly higher than background, most sites, including background, exhibited decreasing concentrations of ${ }^{3} \mathrm{H}$ over timeseven of eleven LANL sites as well as ${ }^{3} \mathrm{H}$ in honey from White Rock/Pajarito Acres and background locations showed significantly ( $p$ $<0.01$ and 0.05 ) decreasing trends (Table 2). The downward trends in ${ }^{3} \mathrm{H}$ concentrations in honey collected from background hives over time were probably a reflection of the relatively short half-life of ${ }^{3} \mathrm{H}$ (12year half-life), weathering, and/or to lower world wide fallout levels that currently exist as a result of the cessation of above ground nuclear testing in the early ' 60 s (Wicker and 
Shultz 1982). The reduction in ${ }^{3} \mathrm{H}$ concentrations in honey over time from LANL and perimeter sites, on the other hand, may be due in part to radioactive decay, weathering, and lower fallout levels, but also to (1) a reduction in ${ }^{3} \mathrm{H}$ operations at LANL, (2) a reduction in air stack emissions and liquid effluents to the environment (EPG 1995), and (3) better engineering controls employed by the Laboratory (Fuehne 1996). These downward trends in ${ }^{3} \mathrm{H}$ concentrations in both bees and honey correlate well with the overall observation of decreasing ${ }^{3} \mathrm{H}$ concentrations in soils within LANL lands over time (Fresquez et al. 1996b).

Tritium concentrations in bees and honey from most LANL and perimeter hive sites were not well correlated with each other (Table 4). Although the analysis of bees and honey for monitoring radioactive constituents in the environment undoubtedly exhibited the presence and distribution of ${ }^{3} \mathrm{H}$
TABLE 4. Pearson's Correlation Coefficients for ${ }^{3} \mathrm{H}$ in bees and honey among LANL, Perimeter, and Regional Background Locations.

\begin{tabular}{lc}
\hline Location & Coefficient \\
\hline LANL & \\
TA-5 & -0.25 \\
TA-8 & -0.19 \\
TA-9 & -0.50 \\
TA-15 & 0.20 \\
TA-16 & -0.24 \\
TA-21 & 0.54 \\
TA-33 & 0.07 \\
TA-35 & 0.06 \\
TA-49 & $0.82^{* *}$ \\
TA-53 & -0.02 \\
TA-54 & 0.70 \\
Perimeter & \\
LA & $0.96^{* *}$ \\
WR/PA & $0.78^{*}$ \\
Background & $0.59^{*}$ \\
\hline
\end{tabular}

${ }^{*}$ and ${ }^{* *}$ denotes a significant correlation between bee and honey at the 0.10 and 0.05 level, respectively.

within the LANL environs, as compared to background, the low correlation between these matrices, especially at sites where ${ }^{3} \mathrm{H}$ was detected in high quantities $\left({ }^{3} \mathrm{H}\right.$ in bees and honey tended to be correlated with each other at sites that contained ${ }^{3} \mathrm{H}$ in lower concentrations like the perimeter and 
background stations), may reflect the complexity of factors related to bee biology/behavior at a given point in time-i.e., the fluctuations in the density of bees, the ratio of foragers to nurse bees collected, longevity, flower/pollen selection, flight activity (light, humidity, and temperature), and water sources and availability, all may have contributed to some degree or another to the lack of correlation between the amount of ${ }^{3} \mathrm{H}$ in bees and the amount of ${ }^{3} \mathrm{H}$ in honey at time of sampling (Drescher 1982, Szabo 1980, Herbert et al. 1970, Woodrow 1968, Rothenbuhler and Kulincevic 1979).

\section{CONCLUSIONS}

Honeybees proved to be useful monitors of ${ }^{3} \mathrm{H}$ presence and distribution in the environment within and around LANL. Although many sites exhibited significantly higher ${ }^{3} \mathrm{H}$ concentrations in bees and honey within and around the LANL environs as compared to background, most sites exhibited decreasing ${ }^{3} \mathrm{H}$ concentrations in bees and honey over time. The amounts of ${ }^{3} \mathrm{H}$ in bees and honey on LANL lands were poorly coorelated with one another.

\section{ACKNOWLEDGMENTS}

Due to funding cutbacks and the fact that honey on LANL lands is not distributed to the public (i.e., not a significant pathway to humans), the program starting in 1996 only included the collection of honey from perimeter areas along the Laboratory-Los Alamos and White Rock/Pajarito Acres townsites-and, background sites. Many thanks to all the people that contributed over the 1979 through 1995 years to this program's success; they included Tom Buhl, Lars Soholt, John Ahlquist, Tom Hakonson, Ken Bostick, Ernie Gladney, Kimber Wallwork-Barber, Gary White, Robert Hayes, Jay Wenzel, Roger Ferenbaugh, and John Salazar. Also, special thanks to Andrea Kron and Kim Nguyen for constructing the figure, and to Tim Haarmann, Roger 
Ferenbaugh and Hector Hinojosa for helpful discussion, review, and editing.

\section{REFERENCES}

Anderson, J.F., and M.A. Wojtas, "Honey Bees (Hymenoptera: Apidae) Contaminated With Pesticides and Polychlorinated Biphenyls," Journal Economic Entomology, 79:12001205 (1986).

Biggs, J., K. Bennett, and P.R. Fresquez, "Radionuclide Contaminant Analysis of Small Mammals at Area G, TA-54, 1994," Los Alamos National Laboratory report LA-13015-MS (1995).

Bromenshenk, J.J., S.R. Carlson, J.C. Simpson, and J.M. Thomas, "Pollution Monitoring of Puget Sound With Honey Bees," Science, 227: 800-801 (1985).

Crane, E., "Bees, Honey, and Pollen as Indicators of Metals in the Environment," Bee World, 65: 47-49 (1984).
Drescher, W., "Suitability of Bees as an Indicator for Environmental Problems," Decheniana-Beihefte (Bonn), 26: 171-177 (1982).

Environmental Protection Group, "Environmental Surveillance at Los Alamos During 1993," Los Alamos National Laboratory report LA12973-ENV (1995).

Environmental Protection Group, "Environmental Surveillance at Los Alamos During 1985," Los Alamos National Laboratory report LA10721-ENV (1986).

Free, J.B., I.H. Williams, A. Townshend, M.S. Basi, and C.L Graham, "Using Foraging Honeybees to Sample an Area for Trace Metals," Environmental International, 9: 9-12 (1983).

Fresquez, P.R., E.L. Vold, and L. Naranjo, Jr., "Radionuclide Concentrations in Vegetation at Radioactive-Waste Disposal Area G During the 1995 Growing Season," 
Los Alamos National Laboratory report LA-13124-PR (1996a).

Fresquez, P.R., M.A. Mullen, J.K. Ferenbaugh, and R.A. Perona, "Radionuclides and Radioactivity in Soils Within and Around Los Alamos National Laboratory, 1974 through 1994: Concentrations, Trends, and Dose Comparisons," Los Alamos National Laboratory report LA-13149-MS (1996b).

Fresquez, P.R., D.A. Armstrong, and L.H. Pratt, "Radionuclide Concentrations in Bees and Honey Within and Around Los Alamos National Laboratory," Journal of Environmenal Science and Health (In press) (1997).

Fuehne, D.P., "Effects of Engineering Controls on Radioactive Air Emissions from the Los Alamos Neutron Science Center," Los Alamos National Laboratory report LA-UR-96-1469 (1996).
Gilbert, R.O., Statistical Methods for Environmental Pollution Monitoring, New York: Van Nostrand Reinhold (1987).

Hakonson, T.E., and K.V. Bostick, "The Availability of Environmental Radioactivity to Honey Bee Colonies at Los Alamos," Journal of Environmental Quality, 5: 307-310 (1976).

Herbert, E.W., W.E. Bickely, and H. Shimanuki, "The Brood-Rearing Capability of Caged Honey Bees Fed Dandelion and Mixed Pollen Diets," Journal of Economic Entomology, 63: 215-218 (1970).

Hollander, M., and D.A Wolfe, Nonparametric Statistical Methods, New York: Wiley, (1973).

Jong, D.D., and R.A. Morse, "Selenium in Pollen Gathered by Bees Foraging on Fly Ash-Grown Plants," Bulletin of Environmental Contamination and Toxicology, 18: 442-444 (1977). 
Kirkham, M.B., and J.C. Corey, "Pollen as Indicator of Radionuclide Pollution," Journal Nuclear Agriculture and Biology, 6: 71-74 (1977).

Morse, R.A., T.W. Culliney, W.H. Gutenmann, C.B. Littman, and D.J. Lisk, "Polychlorinated Biphenyls in Honey Bees," Bulletin of Environmental Contamination Toxicology, 38: 271-276 (1987).

Rothenbuhler, W.C., and J.M. Kulincevic, "Successful Selection of Honeybees for Fast and Slow Hoarding of Sugar Syrup in the Laboratory," Journal of Apicultural Research, 18: 272-278 (1979).

Simmons, M.A., J.J. Bromenshenk, and J.L. Gudatis, "Honeybees as Monitors of Low Levels of Radioactivity," Pacific Northwest Laboratory report PNL-7348/UC-608 (1990).

Szabo, T.I., "Effect of Weather Factors on Honeybee Flight Activity and Colony Weight Gain," Journal of Apicultural Research, 19: 164 171 (1980).

Wallwork-Barber, M.K., R.W. Ferenbaugh, and E.S. Gladney, "The Use of Honey Bees as Monitors of Environmental Pollution," American Bee Journal, 112: 770-772 (1982).

Wicker, W.F., and V. Schultz, Radioecology: Nuclear Energy and the Environment, Boca Raton, FL: CRC Press, Inc., (1982).

Woodrow, A.W., "Some Factors Affecting Selection of Sucrose Solutions by Foraging," American Bee Journal, 313-315 (1968). 